\title{
(6) OPEN ACCESS \\ Meeting the need for corrective spectacles in visually impaired Chinese school children: the potential of ready-made spectacles
}

\author{
Zhuoting Zhu, ${ }^{1}$ Leon B Ellwein, ${ }^{2}$ Sean KWang, ${ }^{3}$ Jialiang Zhao, ${ }^{4}$ Mingguang $\mathrm{He}^{1,5}$
}

\begin{abstract}
- Additional material is published online only. To view please visit the journal online (http://dx.doi.org/10.1136/ bjophthalmol-2018-312262).

${ }^{1}$ State Key Laboratory of Ophthalmology, Zhongshan Ophthalmic Center, Sun Yat-sen University, Guangzhou, China ${ }^{2}$ National Eye Institute, National Institutes of Health, Bethesda, Maryland, USA

Harvard Medical School, Boston, Massachusetts, USA ${ }^{4}$ Chinese Academy of Medical Sciences, Peking Union Medical College Hospital, Beijing, China ${ }^{5}$ Department of Surgery, Centre for Eye Research Australia; Ophthalmology, University of Melbourne, Melbourne, Victoria, Australia
\end{abstract}

\section{Correspondence to}

Dr Mingguang He, Zhongshan Ophthalmic Center, People's Republic of China, Guangzhou 510060, China; mingguanghe@ gmail.com and Professor Jialiang Zhao, Chinese Academy of Medical Sciences, Peking Union Medical College Hospital, Beijing, China; 13501132676@ 163.com

Received 16 March 2018 Revised 8 August 2018 Accepted 30 August 2018 Published Online First 2 October 2018

\begin{abstract}
Purpose To assess the potential of ready-made (spherical) spectacles (RMS) in meeting the need for refractive correction in visually impaired children in China.
\end{abstract}

Methods Eligible children aged 5-17 years were identified from the three study sites in China. Distance visual acuity was measured with a retroilluminated logarithm of the minimum angle of resolution chart with tumbling E optotypes. Cycloplegic autorefraction was performed on all children using a handheld autorefractor. If uncorrected visual acuity (UCVA) was $\leq 20 / 40$ in either eye, best corrected visual acuity was measured with subjective refractive error.

Results A total of 13702 children were enumerated from the three studies, with 12334 (90.0\%) having both reliable visual acuity measurements and successful cycloplegia. Among the 12334 study children, the prevalence of UCVA $\leq 20 / 40$ in the better seeing eye was $16.4 \%$ (95\% Cl $15.0 \%$ to $17.8 \%$ ), with $91.1 \%$ (1843) of these improving by $\geq 3$ lines of visual acuity with refractive correction. Prevalence was 12.7\% (95\% Cl $11.5 \%$ to $13.9 \%$ ) for UCVA $\leq 20 / 50$ with $97.4 \%$ (1521) improving by $\geq 3$ lines, and $9.38 \%(95 \% \mathrm{Cl}$ $8.39 \%$ to $19.4 \%$ ) for UCVA $\leq 20 / 63$ with $98.4 \%$ (1138) improving by $\geq 3$ lines. Depending on the severity of visual impairment, $62.8 \%-64.0 \%$ of children could be accommodated with RMS if not correcting for astigmatism of $\leq 0.75$ dioptres and anisometropia of $\leq 0.50$ spherical equivalent dioptres. Approximately $87 \%$ of children could be accommodated with RMS if astigmatism and anisometropia limits were increased to $\leq 1.25$ and $\leq 1.50$ dioptres, respectively.

Conclusions RMS could substantially alleviate visual morbidity in two-thirds or more of visually impaired schoolchildren in China. This cost-effective approach to refractive correction might also be useful in low/middleincome countries with poor access to optometric services.

\section{INTRODUCTION}

Visual impairment poses substantial public health issues in children. According to the World Health Organization (WHO), approximately 19 million children aged 5-15 years suffer from visual impairment, with nearly 12.8 million cases attributable to uncorrected refractive error (URE) ${ }^{1}$ and half of them residing in China. ${ }^{2}$ The global economic burden of URE has been estimated to be $\$ 269$ billion, ${ }^{3}$ with a disproportionate impact on individuals living in low/middle-income countries. ${ }^{4}$
Refractive error correction has been a priority for the WHO's Vision 2020: The Right to Sight global campaign to eliminate global visual impairment and blindness. ${ }^{6}$ Although the treatment of URE with spectacles is straightforward, high costs and poor access to refractive services limit universal correction. School-based screenings and spectacles distribution programmes have become increasingly common in recent years, ${ }^{7}$ some of which have used ready-made spectacles (RMS) that provide equal spherical correction in each eye $e^{8-10}$ and can be made in bulk at low costs.

In practice, the main limitations excluding RMS as a suitable option for refraction correction are the extents of astigmatism and anisometropia. Studies have postulated that RMS could meet the needs of $66 \%-89 \%$ of adults requiring refractive correction $^{11-13}$ with acceptability in visual function, patient satisfaction and continued use. ${ }^{9}{ }^{10}$ However, little information is available regarding the potential percentage of children with refractive error who could benefit from RMS. Among the studies that have been performed examining RMS in children, the criteria for suitability of RMS have varied, ranging from astigmatism less than 0.75 to 1.25 dioptres (D) and anisometropia less than 0.50 to 2.00 D. $^{9-14}$ The extent to which anisometropia and astigmatism are tolerated also differed considerably among individuals. ${ }^{15}$ To date, there are no reliable estimates of the acceptability of RMS in meeting the need for refractive spectacles under different levels of anisometropia and astigmatism.

This article used cross-sectional data from three study sites in China to examine the suitability of RMS for meeting the need for spectacles in visually impaired children aged 5-17 years.

\section{METHODS}

\section{Study population}

This cross-sectional study used population-based data from two of the original eight survey sites of the Refractive Error Study in Children (RESC) study: Shunyi District, ${ }^{16}$ a semirural area outside of Beijing in northern China, and Guangzhou, ${ }^{17}$ a large metropolis in southern China; as well as school-based data from rural Yangxi County. ${ }^{18}$ For Shunyi and Guangzhou, the study sampling frames were constructed using geographically defined clusters based on local register census data. For Yangxi County, stratified cluster sampling frames were constructed through enumeration of grade-specific classes within 17 county schools. The RESC surveys 
were conducted in children aged 5-15 years with different ethnic origins and environments using a standardised study protocol. The Yangxi County study investigated refractive error and visual impairment in children aged 13-17 years using the standardised RESC protocol. Details of the RESC and Yangxi County enumeration methods have been described elsewhere. ${ }^{16-18}$

The RESC and Yangxi County studies adhered to the tenets of the Declaration of Helsinki. A guardian for each child was informed of the study details and asked to provide signed, informed consent.

\section{Visual acuity and cycloplegic refraction}

Distance visual acuity was measured at $4 \mathrm{~m}$ using a retroilluminated logarithm of the minimum angle of resolution chart with five tumbling E optotypes on each line (Precision Vision, La Salle, Illinois, USA), ranging from -0.30 to 1.00 . Cycloplegia was induced with two drops of $1 \%$ cyclopentolate, administered 5 min apart, with a third drop administered after 20 min. Cycloplegia and pupil dilation were evaluated after an additional 15 min. Cycloplegia was recorded as being present if pupils were dilated over $6 \mathrm{~mm}$ or light reflex was absent. Cycloplegic autorefraction was performed on all children using a handheld autorefractor (ARK-30, Nidek). If uncorrected visual acuity (UCVA) was $\leq 20 / 40$ in either eye, best corrected visual acuity (BCVA) was measured with subjective refraction.

\section{Data analysis}

The prevalence of uncorrected visual impairment was calculated using three levels of severity in the better seeing eye: $\leq 20 / 40$, $\leq 20 / 50$ and $\leq 20 / 63$. The association of age, gender and study site with each of the three levels of vision impairment was investigated with multiple logistic regression.

The need for corrective spectacles was separately defined as either $\geq 2$ or $\geq 3$ lines of visual acuity improvement in the better seeing eye with BCVA compared with that in the better seeing eye with UCVA. If both eyes had the same BCVA, the eye with the smaller magnitude of spherical equivalent refractive error was considered as the better seeing eye. The percentage of visually impaired children needing spectacles was calculated under six screening scenarios: (1) UCVA $\leq 20 / 40$ and $\geq 2$ lines of improvement; (2) UCVA $\leq 20 / 40$ and $\geq 3$ lines of improvement; (3) UCVA $\leq 20 / 50$ and $\geq 2$ lines of improvement; (4) UCVA $\leq 20 / 50$ and $\geq 3$ lines of improvement; (5) UCVA $\leq 20 / 63$ and $\geq 2$ lines of improvement; (6) UCVA $\leq 20 / 63$ and $\geq 3$ lines of improvement.

The potential of RMS in meeting the need for corrective spectacles within each of these six screening scenarios was investigated for a range of specified limits on the acceptability for uncorrected astigmatism and anisometropia. Limits on uncorrected astigmatism were analysed for cylinder values of $\leq 0.75$, $\leq 1.00$ and $\leq 1.50 \mathrm{D}$, and anisometropia was analysed for spherical difference of $\leq 0.50, \leq 1.00$ and $\leq 1.50 \mathrm{D}$. The percentage of children meeting these criteria for each screening scenario was calculated as the ratio of the number of children within acceptable limits to the total number of visually impaired children needing refractive correction.

Statistical analyses were performed using Stata Statistical Software (Stata V.12.0, StataCorp, College Station, TX). CI and $p$ values (significant at the 0.05 level) for prevalence estimates and regression models were calculated with adjustment for clustering effects associated with the sampling design based on a ratio (deff) comparing the actual variance estimate with that obtained through simple random sampling. (Using the Stata svyset command, study sites were declared as strata and clusters as primary sampling units, and estimation commands were adjusted for clustering effects with the Stata svy: prefix.)

\section{RESULTS}

\section{Study population}

A total of 13702 children were enumerated (Shunyi: 6134, Guangzhou: 5053 and Yangxi: 2515), with 12641 (92.3\%) having reliable visual acuity measurements in one or both eyes and an ocular examination. Successful cycloplegic refraction in both eyes was not available for 307 of the examined children, leaving a total of 12334 (90.0\%) children in the study population.

Demographic characteristics of the study population within each of the three study sites are shown in online supplementary table 1. Shunyi and Guangzhou, with children aged 5-15 years, comprised $47.5 \%$ and $35.0 \%$ of the total study sample, respectively. Yangxi, with children aged 13-17 years, comprised 17.5\% of the study sample.

\section{Vision impairment}

The prevalence of UCVA in the better seeing eye by age across all three sites is shown in table 1 . The overall prevalence for the three vision impairment levels, $\leq 20 / 40, \leq 20 / 50$ and $\leq 20 / 63$, was $16.4 \%$ (95\% CI $15.0 \%$ to $17.8 \%$ ), $12.7 \%$ (95\% CI $11.5 \%$ to $13.9 \%$ ) and $9.38 \%$ (95\% CI $8.39 \%$ to $10.4 \%)$, respectively.

In multiple logistic regression modelling with age, gender and study site as covariates, the prevalence of vision impairment was lower in Shunyi compared with Yangxi (the reference site), with statistically significant ORs of $0.62(95 \% \mathrm{CI}$ 0.54 to $0.73, \mathrm{p}<0.001$ ) for UCVA $\leq 20 / 40$ and 0.73 (95\% CI 0.62 to $0.86, \mathrm{p}<0.001)$ for UCVA $\leq 20 / 50$, but not statistically significant for UCVA $\leq 20 / 63$ (OR: $0.84,95 \%$ CI 0.69 to $1.01, \mathrm{p}=0.070$ ). The prevalence of vision impairment in urban Guangzhou was likewise significantly higher compared with Yangxi, with ORs of 2.13 (95\% CI 1.85 to $2.46, \mathrm{p}<0.001$ ), $2.35(95 \%$ CI 2.01 to $2.74, \mathrm{p}<0.001)$ and $2.52(95 \%$ CI 2.13 to $3.00, p<0.001$ ), respectively, for UCVA $\leq 20 / 40, \leq 20 / 50$ and $\leq 20 / 63$. Vision impairment was significantly more prevalent among older subjects with ORs of 1.32 (95\% CI 1.29 to $1.35, \mathrm{p}<0.001)$ for UCVA $\leq 20 / 40,1.40$ (95\% CI 1.37 to 1.45 , $\mathrm{p}<0.001)$ for UCVA $\leq 20 / 50$ and $1.46(95 \%$ CI 1.42 to 1.51 ,

Table 1 Prevalence of uncorrected visual acuity $\leq 20 / 40, \leq 20 / 50$ and $\leq 20 / 63$ in the better seeing eye by age category

\begin{tabular}{lcccccc}
\hline \multirow{2}{*}{$\begin{array}{l}\text { Level of vision } \\
\text { impairment }\end{array}$} & \multicolumn{1}{l}{ Age category (years) } & \multicolumn{1}{l}{$l$} & \\
\cline { 2 - 7 } & 5-6 & $\mathbf{7 - 9}$ & $10-12$ & $13-15$ & $16-17$ & All \\
\hline UCVA $\leq 20 / 40$ & $76(8.96 \%)$ & $143(4.92 \%)$ & $430(11.9 \%)$ & $1220(27.5 \%)$ & $155(29.5 \%)$ & $2024(16.4 \%)$ \\
UCVA $\leq 20 / 50$ & $23(2.71 \%)$ & $89(3.06 \%)$ & $327(9.03 \%)$ & $995(22.4 \%)$ & $128(24.4 \%)$ & $1562(12.7 \%)$ \\
UCVA $\leq 20 / 63$ & $10(1.18 \%)$ & $56(1.93 \%)$ & $226(6.24 \%)$ & $758(17.1 \%)$ & $107(20.4 \%)$ & $1157(9.38 \%)$ \\
\hline
\end{tabular}

Data are given as number (\%) with vision impairment.

UCVA, uncorrected visual acuity. 
Table 2 Improvement of vision impairment in better seeing eye with refractive correction by age category

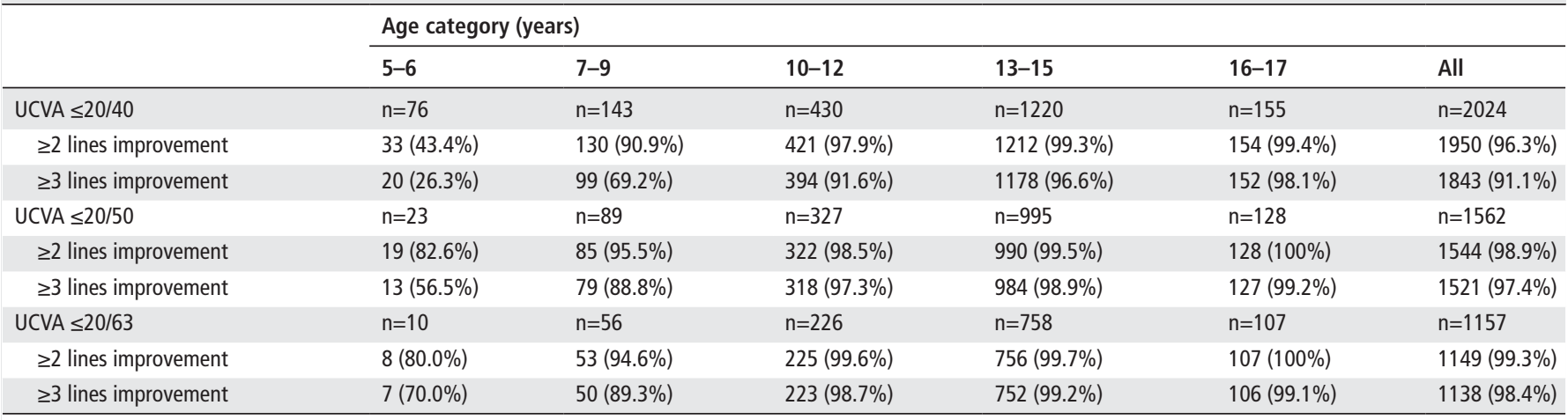

UCVA, uncorrected visual acuity.

$\mathrm{p}<0.001)$ for UCVA $\leq 20 / 63$, and among women with ORs of 1.79 (95\% CI 1.61 to $1.98, \mathrm{p}<0.001), 1.91$ (95\% CI 1.70 to $2.14, \mathrm{p}<0.001)$ and $1.90(95 \%$ CI 1.67 to $2.17, \mathrm{p}<0.001)$, respectively, for UCVA $\leq 20 / 40, \leq 20 / 50$ and $\leq 20 / 63$.

\section{Need for refractive correction}

The need for corrective spectacles in each of the six screening scenarios-based on $\geq 2$ or $\geq 3$ lines of improvement within each of three levels of visual impairment-is shown in table 2. Across all three levels of visual impairment, the need for refractive correction exceeded $90 \%$ by ages $7-9$ years when defined as $\geq 2$ lines of improvement, and by ages 10-12 years when defined as $\geq 3$ lines of improvement. For children aged 5-6 years, the need for refractive correction was most notable for those with UCVA $\leq 20 / 50$ and $\leq 20 / 63$.

\section{Distribution of refractive error}

The distributions of astigmatism with cycloplegic autorefraction in the best corrected, better seeing eye among children with UCVA $\leq 20 / 40, \leq 20 / 50$ or $\leq 20 / 63$ improving by $\geq 2$ or $\geq 3$ lines with refractive correction are shown in figure 1 . The astigmatism median was $0.50 \mathrm{D}$ for all six scenarios, with means and SDs ranging from $0.645 \pm 0.815$ to $0.686 \pm 0.871$ D (online supplementary table 2). The distributions of anisometropia, shown in figure 2 , had a median value of $0.25 \mathrm{D}$ for all six scenarios and means and SDs between $0.418 \pm 0.571$ and $0.466 \pm 0.694 \mathrm{D}$.

\section{Meeting the need with RMS}

The potential of RMS to treat visual impairment is dependent on the maximum amount of astigmatism and anisometropia that can be ignored with a single spherical-only correction. Percentages of astigmatism and anisometropia within specified limits for each of the six screening scenarios are shown in table 3. Children with astigmatism $\leq 0.75 \mathrm{D}$ and with anisometropia $\leq 0.50 \mathrm{D}$ represent reasonable candidates for RMS based on WHO recommendation. ${ }^{14}$ The percentage of children with these minimal amounts of astigmatism and
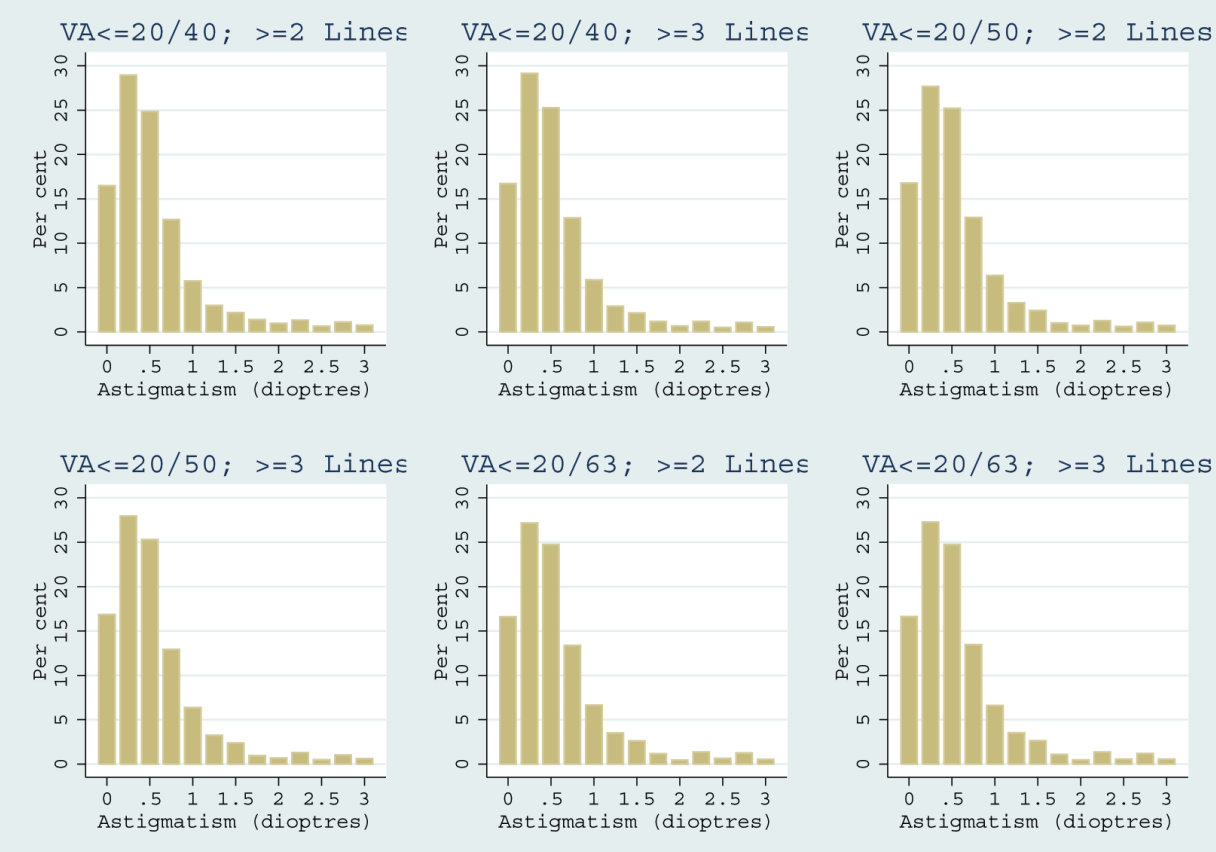

Figure 1 The distributions of astigmatism measured with cycloplegic autorefraction in visually impaired children improving by $\geq 2$ or $\geq 3$ lines with refractive correction in the best corrected, better seeing eye. VA, visual acuity. 

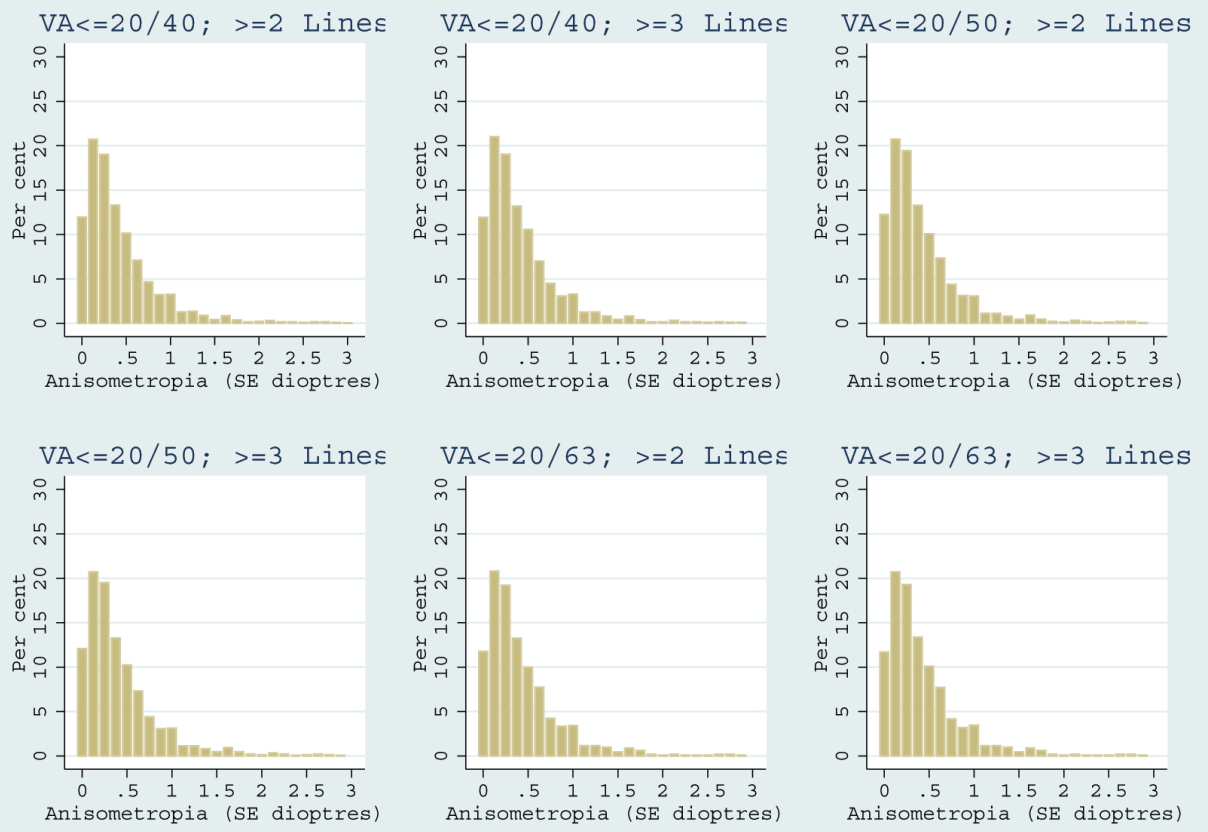

Figure 2 The distributions of spherical equivalent anisometropia measured with cycloplegic autorefraction in visually impaired children improving by $\geq 2$ or $\geq 3$ lines with refractive correction in the best corrected, better seeing eye. SE, spherical equivalent; VA, visual acuity.

Table 3 Children suitable for RMS in six scenarios based on uncorrected visual acuity, improvement of visual impairment, astigmatism and anisometropia

\begin{tabular}{|c|c|c|c|c|}
\hline \multirow[b]{2}{*}{ Astigmatism category (D) } & \multicolumn{4}{|c|}{ Anisometropia category (D) } \\
\hline & $\leq 0.50$ & $\leq 1.00$ & $\leq 1.50$ & Unlimited \\
\hline$\leq 0.75$ & $\begin{array}{l}\text { SC1: } 1217(62.4 \%) \\
\text { SC2: } 1179(64.0 \%) \\
\text { SC3: } 975(63.2 \%) \\
\text { SC4: } 968(63.6 \%) \\
\text { SC5: } 718(62.5 \%) \\
\text { SC6: } 715(62.8 \%)\end{array}$ & $\begin{array}{l}\text { SC1: } 1473(75.5 \%) \\
\text { SC2: } 1419(77.0 \%) \\
\text { SC3: } 1167(75.6 \%) \\
\text { SC4: } 1159(76.2 \%) \\
\text { SC5: } 864(75.2 \%) \\
\text { SC6: } 860(75.6 \%)\end{array}$ & $\begin{array}{l}\text { SC1: } 1519(77.9 \%) \\
\text { SC2: } 1464(79.4 \%) \\
\text { SC3: } 1202(77.9 \%) \\
\text { SC4: } 1194(78.5 \%) \\
\text { SC5: } 892(77.6 \%) \\
\text { SC6: } 888(78.0 \%)\end{array}$ & $\begin{array}{l}\text { SC1: } 1559(80.0 \%) \\
\text { SC2: } 1502(81.5 \%) \\
\text { SC3: } 1235(80.0 \%) \\
\text { SC4: } 1227(80.7 \%) \\
\text { SC5: } 913(79.5 \%) \\
\text { SC6: } 909(79.9 \%)\end{array}$ \\
\hline$\leq 1.00$ & $\begin{array}{l}\text { SC1: } 1288(66.1 \%) \\
\text { SC2: } 1248(67.7 \%) \\
\text { SC3: } 1039(67.3 \%) \\
\text { SC4: } 1031(67.8 \%) \\
\text { SC5: } 767(66.8 \%) \\
\text { SC6: } 763(67.1 \%)\end{array}$ & $\begin{array}{l}\text { SC1: } 1570(80.5 \%) \\
\text { SC2: } 1514(82.2 \%) \\
\text { SC3: } 1254(81.2 \%) \\
\text { SC4: } 1245(81.9 \%) \\
\text { SC5: } 933(81.2 \%) \\
\text { SC6: } 928(81.6 \%)\end{array}$ & $\begin{array}{l}\text { SC1: } 1620(83.1 \%) \\
\text { SC2: } 1562(84.8 \%) \\
\text { SC3: } 1291(83.6 \%) \\
\text { SC4: } 1282(84.3 \%) \\
\text { SC5: } 961(83.6 \%) \\
\text { SC6: } 956(84.0 \%)\end{array}$ & $\begin{array}{l}\text { SC1: } 1667(85.5 \%) \\
\text { SC2: } 1607(87.2 \%) \\
\text { SC3: } 1330(86.1 \%) \\
\text { SC4: } 1321(86.9 \%) \\
\text { SC5: } 987(85.9 \%) \\
\text { SC6: } 982(86.3 \%)\end{array}$ \\
\hline$\leq 1.25$ & $\begin{array}{l}\text { SC1: } 1322(67.8 \%) \\
\text { SC2: } 1280(69.5 \%) \\
\text { SC3: } 1070(69.3 \%) \\
\text { SC4: } 1061(69.8 \%) \\
\text { SC5: } 793(69.0 \%) \\
\text { SC6: } 789(69.3 \%)\end{array}$ & $\begin{array}{l}\text { SC1: } 1618(83.0 \%) \\
\text { SC2: } 1559(84.6 \%) \\
\text { SC3: } 1297(84.0 \%) \\
\text { SC4: } 1287(84.6 \%) \\
\text { SC5: } 969(84.3 \%) \\
\text { SC6: } 964(84.7 \%)\end{array}$ & $\begin{array}{l}\text { SC1: } 1673(85.8 \%) \\
\text { SC2: } 1611(87.4 \%) \\
\text { SC3: } 1337(86.6 \%) \\
\text { SC4: } 1327(87.3 \%) \\
\text { SC5: } 999(87.0 \%) \\
\text { SC6: } 994(87.4 \%)\end{array}$ & $\begin{array}{l}\text { SC1: } 1723(88.4 \%) \\
\text { SC2: } 1659(90.0 \%) \\
\text { SC3: } 1379(89.3 \%) \\
\text { SC4: } 1369(90.0 \%) \\
\text { SC5: } 1026(89.3 \%) \\
\text { SC6: } 1021(89.7 \%)\end{array}$ \\
\hline Unlimited & $\begin{array}{l}\text { SC1: } 1451(74.4 \%) \\
\text { SC2: } 1384(75.1 \%) \\
\text { SC3: } 1158(75.0 \%) \\
\text { SC4: } 1142(75.1 \%) \\
\text { SC5: } 855(74.4 \%) \\
\text { SC6: } 849(74.6 \%)\end{array}$ & $\begin{array}{l}\text { SC1: } 1803(92.5 \%) \\
\text { SC2: } 1710(92.8 \%) \\
\text { SC3: } 1432(92.8 \%) \\
\text { SC4: } 1411(92.8 \%) \\
\text { SC5: } 1068(93.0 \%) \\
\text { SC6: } 1058(93.0 \%)\end{array}$ & $\begin{array}{l}\text { SC1: } 1879(96.4 \%) \\
\text { SC2: } 1779(96.5 \%) \\
\text { SC3: } 1485(96.2 \%) \\
\text { SC4: } 1464(96.3 \%) \\
\text { SC5: } 1110(96.6 \%) \\
\text { SC6: } 1100(96.7 \%)\end{array}$ & $\begin{array}{l}\text { SC1: } 1950(100 \%) \\
\text { SC2: } 1843(100 \%) \\
\text { SC3: } 1544(100 \%) \\
\text { SC4: } 1521(100 \%) \\
\text { SC5: } 1149(100 \%) \\
\text { SC6: } 1138(100 \%)\end{array}$ \\
\hline
\end{tabular}

SC1 ( $n=1950):$ UCVA $\leq 20 / 40$ improving by $\geq 2$ lines.

$S C 2$ ( $n=1843)$ : UCVA $\leq 20 / 40$ improving by $\geq 3$ lines.

SC3 ( $n=1544):$ UCVA $\leq 20 / 50$ improving by $\geq 2$ lines.

SC4 ( $n=1521):$ UCVA $\leq 20 / 50$ improving by $\geq 3$ lines.

SC5 ( $n=1149):$ UCVA $\leq 20 / 63$ improving by $\geq 2$ lines.

SC6 ( $n=1138$ ): UCVA $\leq 20 / 63$ improving by $\geq 3$ lines.

Data are given as number (\%) within astigmatism and anisometropia categories for each of the UCVA and lines of improvement scenarios: SC1-SC6.

RMS, ready-made spectacles; UCVA, uncorrected visual acuity. 
anisometropia ranged from $62.4 \%$ to $64.0 \%$ across the six screening scenarios. In contrast, when criteria for RMS were expanded to astigmatism $\leq 1.25 \mathrm{D}$ and anisometropia $\leq 1.50$ $\mathrm{D}$, the percentage of children within these bounds ranged from $85.8 \%$ to $87.4 \%$.

If RMS could include the possibility of a spherical power for the fellow eye different from that for the better seeing eye, then the amount of anisometropia would not be important, and the percentage of children that could be accommodated with RMS would range from $79.5 \%$ to $90.0 \%$ depending on astigmatism limits.

\section{DISCUSSION}

To our knowledge, this was the first study to investigate the potential of RMS in meeting the need for refractive correction in visually impaired Chinese schoolchildren under different criteria of anisometropia and astigmatism. In our study population, upward of two-thirds of children in need of refractive correction could be accommodated with single-sphere RMS.

In our analysis, the need for refractive correction with at least two or three lines of improvement ranged from $91.1 \%$ to $99.3 \%$ among Chinese schoolchildren. However, this percentage was only $26.3 \%$ for $\geq 2$ lines and $43.4 \%$ for $\geq 3$ lines of improvement among children aged 5-6 years with UCVA $\leq 20 / 40$. These differences for 5 and 6 year-old children are to be expected, as the visual and cognitive systems at this age range are still developing. Moreover, it has been shown that among Chinese preschoolers aged 5 and 6 years, the single-sided 95 th percentile of the emmetropia children occurs at a UCVA of 20/40. ${ }^{19}$

High prevalence of URE among children has been reported both in low/middle-income countries and developed countries. ${ }^{20}$ The advantages of providing RMS to alleviate URE among children include both financial and practical reasons. Good-quality RMS are substantially cheap (US $\$ 0.50$ per pair of spectacles) ${ }^{8}$ when purchased in volume and RMS can be provided at the time of refraction, thereby reducing distribution costs. Nevertheless, because RMS prescription is typically based on the eye with lower refractive error, the fellow eye may be undercorrected and have slightly worse visual acuity. Another downside of RMS is that children with astigmatism are not given cylindrical correction. Although two randomised controlled trials conducted in India ${ }^{9}$ and $\mathrm{China}^{10}$ found statistically significant differences in corrected visual acuity between groups receiving customised spectacles versus RMS, most of these differences only affected one eye and accounted for less than one line of Snellen acuity. Moreover, differences in corrected visual acuity between the customised spectacles and RMS groups did not translate into differences in acceptability or values in terms of visual function, patient satisfaction or continued use.

For the majority of children, RMS should be able to meet refractive needs. However, some studies have speculated above certain limits of anisometropia $(0.50-2.00 \mathrm{D})$ or astigmatism $(0.75-1.25 \mathrm{D})$, spherical correction with RMS is no longer appropriate. ${ }^{11-13}$ Previous studies have found that $66 \%-89 \%$ of adults needing refractive correction could be accommodated with single-sphere RMS. ${ }^{11-13}$ Our study instead explored the potential of RMS among visually impaired schoolchildren in need of refractive correction. Instead of using an arbitrary definition of suitable candidates for RMS, we explored the feasibility of RMS under six scenarios combining different levels of astigmatism $(0.75-1.25 \mathrm{D})$ and anisometropia $(0.50-1.50 \mathrm{D}) .^{9-14}$ Although one prior study found a negative linear relationship between degrees of astigmatism or anisometropia and RMS satisfaction, ${ }^{9}$ the extent to which anisometropia and astigmatism are tolerated varied considerably among individuals. ${ }^{15}$ Based on this, we concluded that completely excluding children according to arbitrary anisometropia or astigmatism cut-offs may remove potential beneficiaries of RMS.

Since school-based screenings and provision of spectacles are key global strategies to reduce avoidable blindness and visual impairment, ${ }^{7}$ our main finding has important implications for planners of public health programmes in that more than two-thirds of visually impaired Chinese schoolchildren in need of refractive correction could potentially benefit from RMS. We recommend four steps for school-based programmes to implement RMS. First, visual acuity and refraction screening should identify children who could benefit from spectacles provision. Second, RMS should be obtained, either from local or external mass production or donations. Third, RMS should be initially trialled on all children in need of refractive correction, from which children themselves could help decide if upgrading to customised spectacles is worthwhile. Finally, regular follow-up care should be implemented, including spectacles repair and additional dispensing. All of these steps could be realised by paramedical staff with basic training in subjective refraction. Our analysis may also be of practical application in other communities, especially in countries and sites with high rates of refractive error and where eye care services may be limited. The efficacy of RMS depends on the characteristics of the population requiring refractive correction. Consistent evidence has showed that URE is the leading cause of visual impairment among schoolchildren. ${ }^{14-1619}$ Furthermore, multicountry studies have revealed relatively low prevalence rates for astigmatism and anisometropia, with only $13.3 \%$ of $5-15$ year-old children having astigmatism $\geq 0.75 \mathrm{D}$ in magnitude and 6\% of 6-18 year-old children having anisometropia $\geq 1.00 \mathrm{D},{ }^{21}$ implying the potential of RMS to be used in other settings.

The advantages of our analysis include large sample sizes and high response rates. However, some of our results should be interpreted with cautions. As our study was cross-sectional in design and used data sets collected almost 20 years ago, it is possible that the rates of astigmatism and anisometropia that we calculated may not fully reflect current trends. Nonetheless, a recent meta-analysis showed that prevalence rates for astigmatism and anisometropia have remained low and relatively constant over time. ${ }^{22}$ Additionally, although the Shunyi and Guangzhou surveys were population-based, a school-based sampling strategy was used in Yangxi County, and therefore the Yangxi survey may not be fully representative of the entire county population. However, the aim of our study is to theoretically show the effectiveness of RMS for refractive correction using this large test sample. We do not aim at advocating to use RMS in developed areas where majority of children have already had customised spectacles, such as Guangzhou. Furthermore, given the strong association between myopia and socioeconomic factors (eg, education level), myopia is more prevalent in those with a privilege socioeconomic background, who are more likely to have access to the refractive correction. Nevertheless, our results provide insights of the potential efficiency of RMS in meeting the need for refractive correction in visually impaired schoolchildren, which could be useful in low-resource areas with poor access to optometric services and prescription spectacles.

In summary, RMS could substantially alleviate visual morbidity in two-thirds or more of visually impaired schoolchildren in need of corrective spectacles. Incorporating RMS into vision screening programmes and optometry services may enable us to 
realistically achieve the goal of the WHO's Vision 2020 initiative, especially in low-resource areas.

Contributors Conception and design (JZ, MH); data collection (JZ, MH); analysis and interpretation (ZZ, LBE, SKW, JZ, MH); writing of the article (ZZ, LBE); critical revision of the article (SKW, JZ, MH).

Funding The present work was supported by the World Health Organization under National Institutes of Health contract N01-EY-2103, the Fundamental Research Funds of the State Key Laboratory in Ophthalmology, and the National Natural Science Foundation of China (81125007). MH receives support from the University of Melbourne at Research Accelerator Program and the CERA Foundation. The Centre for Eye Research Australia receives Operational Infrastructure Support from the Victorian State Government.

Disclaimer The sponsor or funding organisation had no role in the design or conduct of this research.

Competing interests None declared.

Patient consent Parental/guardian consent obtained.

Ethics approval WHO Secretariat Committee on Research Involving Human Subjects.

Provenance and peer review Not commissioned; externally peer reviewed.

Open access This is an open access article distributed in accordance with the Creative Commons Attribution Non Commercial (CC BY-NC 4.0) license, which permits others to distribute, remix, adapt, build upon this work non-commercially, and license their derivative works on different terms, provided the original work is properly cited, appropriate credit is given, any changes made indicated, and the use is non-commercial. See: http://creativecommons.org/licenses/by-nc/4.0/

\section{REFERENCES}

1. World Health Organization, 2016. Visual impairment and blindness (2014). http:// www.who.int/mediacentre/factsheets/fs282/en/ (accessed 3 Feb 2016).

2. Resnikoff S, Pascolini D, Mariotti SP, et al. Global magnitude of visual impairment caused by uncorrected refractive errors in 2004. Bull World Health Organ 2008;86:63-70

3. Smith TS, Frick KD, Holden BA, et al. Potential lost productivity resulting from the global burden of uncorrected refractive error. Bull World Health Organ 2009;87:431-7.

4. Chandra SR. Global blindness: VISION 2020: the right to sight. Arch Ophthalmol 2008;126:1457.
5. Naidoo KS, Wallace DB, Holden BA, et al. The challenge of uncorrected refractive error: driving the agenda of the Durban Declaration on refractive error and service development. Clin Exp Optom 2010;93:131-6.

6. Dandona L, Dandona R. What is the global burden of visual impairment? BMC Med 2006;4.

7. Sharma A, Congdon N, Patel M, et al. School-based approaches to the correction of refractive error in children. Surv Ophthalmol 2012;57:272-83.

8. Ramke J, Palagyi A, Toit $R$, et al. Applying standards to readymade spectacles used in low-resource countries. Optom Vis Sci 2009;86:1104-11.

9. Keay L, Gandhi M, Brady C, et al. A randomized clinical trial to evaluate ready-made spectacles in an adult population in India. Int J Epidemiol 2010;39:877-88.

10. Zeng Y, Keay L, He M, et al. A randomized, clinical trial evaluating ready-made and custom spectacles delivered via a school-based screening program in China. Ophthalmology 2009;116:1839-45.

11. Bourne RR, Dineen BP, Huq DM, et al. Correction of refractive error in the adult population of Bangladesh: meeting the unmet need. Invest Ophthalmol Vis Sci 2004;45:410-7.

12. Maini $R$, Keeffe J, Weih LA, et al. Correction of refractive error in the Victorian population: the feasibility of "off the shelf" spectacles. Br J Ophthalmol 2001;85:1283-6.

13. Fledelius HC. Prevalences of astigmatism and anisometropia in adult danes. With reference to presbyopes' possible use of supermarket standard glasses. Acta Ophthalmol 1984;62:391-400.

14. WHO. Elimination of avoidable visual disability due to refractive errors. Report of an informal planning meeting. Prevention of Blindness and Deafness. Geneva, 2000.

15. du Toit R, Ramke J, Brian G. Tolerance to prism induced by readymade spectacles: setting and using a standard. Optom Vis Sci 2007;84:1053-9.

16. Zhao J, Pan X, Sui R, et al. Refractive error study in children: results from Shunyi District, China. Am J Ophthalmol 2000;129:427-35.

17. He M, Zeng J, Liu Y, et al. Refractive error and visual impairment in urban children in southern china. Invest Ophthalmol Vis Sci 2004;45:793-9.

18. He $M$, Huang $W$, Zheng $Y$, et al. Refractive error and visual impairment in school children in rural southern China. Ophthalmology 2007;114:374-82.

19. Guo X, Fu M, Lu J, et al. Normative distribution of visual acuity in 3- to 6-year-old Chinese preschoolers: the Shenzhen kindergarten eye study. Invest Ophthalmol Vis Sci 2015;56:1985-92.

20. Murthy GV, Gupta SK, Ellwein LB, et al. Refractive error in children in an urban population in New Delhi. Invest Ophthalmol Vis Sci 2002;43:623-31.

21. Czepita D, Gosławski W, Mojsa A. Occurrence of anisometropia among students ranging from 6 to 18 years of age. Klin Oczna 2005;107:297-9.

22. Hashemi H, Fotouhi A, Yekta A, et al. Global and regional estimates of prevalence of refractive errors: systematic review and meta-analysis. J Curr Ophthalmol 2018;30:3-22 